\author{
Rajagopal Appavu* and Deepa Mohan \\ Department of Molecular Biophysics Unit, Indian \\ Institute of Science, Bangalore, India
}

Dates: Received: 29 February, 2016; Accepted: 07 March, 2016; Published: 08 March, 2016

${ }^{*}$ Corresponding author: Rajagopal Appavu, Department of Molecular Biophysics Unit, Indian Institute of Science, Bangalore, 560012, E-mail: dominiquerajagopal@gmail.com; arg@mbu.iisc.ernet.in www.peertechz.com

Keywords: 26S Proteasome; Multiple myeloma; Bortezomib; Inhibition of transcriptional factors: Apoptosis

\author{
Mini Review
}

\section{Bortezomib in Anti-Cancer Activity: A Potential Drug}

\section{Multiple Myeloma (MM)}

Multiple myeloma is an incurable malignant B-cell tumor, suffers from diminished immune system. Plasma cell, white blood cells significantly produces infection fighting antibodies. When cancer occurs, the body produces plasma cells uncontrollably. These abnormal plasma cells accumulate in bone marrow and solid parts of the bone and these cells are called myeloma cells. When myeloma cells form tumors in multiple areas of bone called multiple myeloma. Chemotherapy, radiation therapy and stem cell transplantation are the treatments for multiple myeloma to increase survival but the disease remains not curable [1].

Proteasome is barrel shaped, multicatalytic, enzymatic complex present in both cytoplasm and nucleus of all eukaryotic cells responsible for the targeted degradation of proteins which are involved in many cellular process. These process mediated by ubiquitin conjugating system requires ubiquitin activating E1 enzyme, E2 conjugating enzyme, and E3 ubiquitin ligase. Ubiquitin is activated by formation of thioester bond with E1, then ubiquitin is transferred to ubiquitin conjugating enzyme E2. Finally, peptide bond is formed between C-terminal carboxylate residue of ubiquitin and lysine residue of targeted proteins. A number of ubiquitin ligase binds to the substrate resulting in polyubiquitination. The polyubiquitin chain enters into proteasome degraded into small peptides [2,3]

ATP dependent, 26S proteasome consists of $20 \mathrm{~S}$ proteasome core particle, capped at each end by $19 \mathrm{~S}$ regulatory particle. $20 \mathrm{~S}$ proteasome is cylindrical shaped, composed of 4-stacked rings. The top and bottom ring formed by seven $\alpha$-subunits and the two inner cores formed by seven $\beta$-subunits, protease active sites. The subunits of $\beta 1,2$, and $\beta 5$ contain postglutamyl peptidyl hydrolytic- like activity preferably cleave after acidic amino acids, tryptic-like activity preferably cleave after basic amino acids, chymotryptic-like activity preferably cleave after hydrophobic amino acids, respectively [4]. 19S regulatory particle consists of lid and base which is used to remove ubiquitin tags from targeted protein, feed them into inner catalytic site of $20 \mathrm{~S}$ core particle [5]. Proteasome targets cell cycle regulators, tumor suppressors, transcription factors, signaling molecules, mutants, and damaged proteins. Disruption of these molecules by proteasome inhibition results in cell cycle arrest or cell death [6].

\section{Proteasome inhibitors}

A variety of natural and synthetic proteasome inhibitors are available such as peptide aldehydes, peptide a-ketoaldehydes, peptide vinyl sulfones, lactacystin, epoxomicin, peptide macrocycles, $\gamma$-lactam thiol ester, epipolythio-dioxopiperazine toxin and eponemycin. But many of these inhibitors are lack in specificity, poor metabolic stability, fast dissociation rate, irreversible and low affinity of binding makes them less suitable for use. For example, peptide aldehyde inhibitors dissociate rapidly and inactivated by oxidization which allows only short-lived proteasome inhibition. These problems overcome by replacing the corresponding aldehyde group in peptides by boronic acid [2]. Because it is forming stable tetrahedral transition state intermediate with $\mathrm{N}$-terminal threonine residue of $\beta 5$ active site and also peptide boranates dissociate more slowly and attaining stable inhibition [7].

\section{Bortezomib}

Bortezomib (Velcade ${ }^{\circledR}$ PS-341, MG-341, MLN-431 and Millennium Pharmaceuticals, Inc.) is a modified dipeptidyl boronic acid, written as $\mathrm{N}$-pyrazinecarbonyl-L-phenylalanine- $\mathrm{L}$ leucine boronic acid with the molecular formula of $\mathrm{C}_{19} \mathrm{H}_{25} \mathrm{BN}_{4} \mathrm{O}_{4}$ and the molecular mass of $384.24 \mathrm{Da}$. It is the first, selective and only proteasome inhibitor for the treatment of relapsed and refractory multiple myeloma has been approved by U.S. Food and Drug Administration (FDA) in 2003. It is also investigated for the treatment of other malignancies such as non-Hodgkin's lymphoma, solid tumors, prostate, breast, ovarian, colorectal, pancreatic, and non-small-cell lung cancers [4]. In combination therapy, bortezomib induce chemo-/radio-sensitization or reduce drug resistance. It is a small, water soluble, more potent, very high affinity, selective, highly specific and reversible proteasome inhibitor, in addition of low molecular weight and easy of synthesis [8]. 
<smiles>CC(C)C[C@H](NC(=O)[C@H](Cc1ccccc1)NC(=O)c1cnccn1)B(O)O</smiles>

Bortezomib is designed according to fit in active site of the proteasome. It is a quite remarkable drug for cancer therapeutics due to the presence of empty p-orbital on boron atom. It can accommodate lone pair of electrons form oxygen atom of threonine, present in chymotrypsin like active site of $\beta 5$ subunit of $20 \mathrm{~S}$ proteasome. Boron atom forms a stable and reversible $\mathrm{sp}^{3}$ hybridized tetrahedral intermediate complex, allows more solubility, membrane permeability, dissociates slowly and stable inhibition of proteasome [9]. This pathway allowed to inhibit degradation of proteins, essential for cell proliferation and survival. Disruption of this degradation arrests cell growth and leads to apoptosis. Bortezomib involved in inhibition of NF- $k$ B activation, induction of apoptosis, blocking of cell-cycle progression, and in anti-angiogenesis [10].

NF- $k$ B is Nuclear Factor Kappa-light chain-enhancer of activated $\mathrm{B}$ cells, a protein involved in transcription of DNA, cell survival, cell signaling, immune and inflammatory response. There are five NF$k \mathrm{~B}$ proteins are present such as RelA/NF $k \mathrm{~B}-\mathrm{p} 65$, RelB, c-Rel, NF$k \mathrm{~B} 1 / \mathrm{NF} k \mathrm{~B}-\mathrm{p} 105$, and NF- $k \mathrm{~B} 2 / \mathrm{NF} k \mathrm{~B}-\mathrm{p} 100$. Each of them activates their own characteristic set of genes. In cytoplasm of all eukaryotic cells, NF- $k \mathrm{~B} 1$ and NF- $k \mathrm{~B} 2$ are synthesized as precursors of p105 and p100, which undergo proteasome degradation to produce mature $\mathrm{NF}-k \mathrm{~B}$ with the subunits of $\mathrm{p} 50$ and $\mathrm{p} 52$. NF- $k \mathrm{~B}$ is inhibited by its inhibitor of $\mathrm{I} k \mathrm{~B}$ (Inhibitor of $\boldsymbol{k B}$ ). Seven different $\mathrm{I} k \mathrm{~B}$ are present such as $\mathrm{I} k \mathrm{~B}$-alpha, I $k \mathrm{~B}$-beta, I $k \mathrm{~B}$-gamma, I $k \mathrm{~B}$-epsilon, Bcl-3, p100 and p105. External stimulation by chemotherapy, radiation, viruses, cytokines, antigens, oxidants and stress initiate binding of cytokines to their surface receptors. I $k \mathrm{~B}$ kinase, a heterodimeric protein kinase activates the phosphorylation on two serine residues $\left(\mathrm{Ser}^{32}\right.$ and $\mathrm{Ser}^{36}$ ) of $\mathrm{I} k \mathrm{~B}$. Phophorylated $\mathrm{I} k \mathrm{~B}$ is recognized by E3RS, an E3 ubiquitin ligase initiating ubiquitination. Ubiquitinated $\mathrm{I} k \mathrm{~B}$ is degradated by $26 \mathrm{~S}$ proteasome with the release of active and mature NF- $k \mathrm{~B}$ which translocates into the nucleus and activates transcription factors, angiogenesis factors, anti-apoptosis, expression of cell-adhesion molecules, chemokines, cyclin $\mathrm{D}$, various cytokines and their receptors. These factors promote cell growth and protect cell from apoptosis [11].

Bortezomib blocks the phosphorylation and degradation of $\mathrm{I} k \mathrm{~B}$ by inhibits $26 \mathrm{~S}$ proteasome. It leads to protect the release of mature $\mathrm{NF}-k \mathrm{~B}$ which decreases the expression of $\mathrm{Bcl}-\mathrm{xL}$ (involved in cancer cell survival) and promotes to apoptosis.

\section{Inhibition of cell adhesion molecules}

Adhesion of Multiple myeloma cells to bone marrow stromal cells (BMSCs) promotes NF- $k \mathrm{~B}$ dependant transcription and secretion of InterLeukin-6 (IL-6), involved in tumor cell growth. This process provides anti-apoptosis, tumor cell survival and chemotherapeutic resistance. The mechanism of chemotherapeutic resistance is not clear. Inhibition of NF- $k$ B activation blocks the secretion of IL-6 and leads to apoptosis. Proteasome inhibition by Bortezomib downregulates the adhesion of Multiple myeloma cells to BMSCs, decreases the cancer cells to chemotherapeutic resistance [12].

In multiple myeloma, NF- $k \mathrm{~B}$ activation increases the expression of cell adhesion molecules such as ICAM-1 (inter cellular adhesion molecules- 1 or cluster of differentiation 54 or CD-54) and VCAM-1 (vascular cell adhesion molecule-1 or cluster of differentiation 106 or CD-106). These adhesion molecules are involved in the binding of multiple myeloma cells to bone marrow stromal cells achieves antiapoptosis. Proteasome inhibition decreases the expression of these adhesion molecules and achieves apoptosis [4,5].

\section{Inhibition of angiogenesis}

Angiogenesis is the formation of new blood vessels, which are provide oxygen and nutrients to cancer cells, invade and spread into other parts of the body, form new cancer cells. Tumor cells produce various molecules to enhance the angiogenesis. Among them, two activators are more important such as basic fibroblast growth factor (bFGF), vascular endothelial growth factor (VEGF). These proteins bind on the corresponding receptors of bFGFR and VEGFR which are present on tumor associated blood vessels. After binding, activates signal into nucleus and promotes a group of genes to make new blood vessel.

NF- $k \mathrm{~B}$ activation induces the expression of various cytokines which are involved in angiogenesis. Bortezomib inhibits the NF- $k \mathrm{~B}$ activation leads to anti-angiogenesis $[2,13]$.

\section{Inhibition of cell-cycle progression}

Cyclin is a short lived regulatory protein, activates cyclindependent kinases (CDKs) to induce cell cycle progression. Different cyclins act at different stages of cell cycle such as cyclin $\mathrm{D}$ and cyclin $\mathrm{E}$ are involved in G1 phase; cyclin E and cyclin A are involved in S phase; cyclin A and cyclin B are involved in mitosis phase; Furthermore, CDC phosphatase is an enzyme, required to dephosphorylate the cyclin-dependant kinases (CDKs) and binding of cyclin at each cycle. Elaborately, cyclin D is the first cyclin produced in cell cycle, binds to CDK4 forming an active complex of cyclin $\mathrm{D}$-CDK4. This complex phosphorylates retinoblastoma $(\mathrm{Rb})$ protein. The hypophosphorylated retinoblastoma dissociates from E2F$\mathrm{Rb}$ complex and activating E2F. The activated E2F codifies various transcription factors including cyclin $\mathrm{E}$ and cyclin $\mathrm{A}$. The produced cyclin E binds to CDK2, forming the complex of cyclin E-CDK2. This complex promotes the cell from G1 phase to $\mathrm{S}$ phase. Cyclin B binds with $\mathrm{CDC} 2$, forming cyclin $\mathrm{B}-\mathrm{CDC} 2$ complex which promotes the cell from G2 phase to mitosis phase, breakdown the nucleus envelope and initiates prophase. This pathway leads to cell cycle progression.

In each cycle, cyclin, CDKs, and CDC enzyme has to degrade by proteasome to allow another phase to progress. Inhibition of proteasome by Bortezomib may arrest the cell cycle by blocking the degradation of cyclin, CDKs, and CDC enzyme.

\section{Inhibition of p53}

p53 is a tumor suppressor protein with the molecular mass of 
$53 \mathrm{KDa}$, involved in the regulation of cell cycle, DNA repair, and apoptosis. In cell cycle, activated p53 binds with DNA, produces various proteins including $\mathrm{p} 21$ which is a cyclin-dependent kinase inhibitor-1. The p21 binds with G1-S/CDK and S/CDK complexes and inhibit their action on cell cycle. As a result, the cell cannot able to proceed to the next stage of cell cycle, leads to apoptosis.

External and Internal stimulation of chemical- or radiation induced DNA breakage, viruses hypoxia, and oncogene activation induces mutations in p53. The mutated p53 cannot able to bind with DNA and produces $\mathrm{p} 21$. As a result, the cell divides uncontrollably and leads to tumor formation. MDM2 is a protein, degraded from p53. In tumor cell, MDM2 binds with mutated p53 and prevents its action by exporting them from nucleus to cytoplasm where mutated p53 can be degraded by $26 \mathrm{~S}$ proteasome [4].

In normal cell, p53 has to be stable in the nucleus to progress apoptosis. It can be achieved by inhibiting the binding of MDM2 on p53. There are two ways to promote such as phosphorylation of p53 inhibits the binding to MDM2; and binding of ARF, a tumor suppressor gene on MDM2 which inhibits MDM2 action as ubiquitin E3 ligase [14].

Recent results show that bortezomib induces the binding of p53 on DNA, promotes transcription factors and inhibits MDM2 [15].

\section{Combination therapy}

Patients who are not responding for bortezomib as a single agent has been responded by combine with other drugs. Bortezomib combines with melphalan is a promising good results for multiple myeloma. Pegylated doxorubicin combined with bortezomib is also used for MM. In solid tumors, bortezomib combined with chemotherapeutic agents of gemcitabine, doxorubicin, irinotecan, docetaxel and paclitaxel are producing significant results in phase I trials [16].

\section{Side effects of bortezomib}

Bortezomib has been administrated as cyclical therapy, requires twice weekly. For multiple myeloma, 13 cycles are needed. But the continues dosing of bortezomib produces dose-limiting toxicities, drug resistance, interference by natural compounds [17] and has few side effects such as weakness, nausea, tiredness, trouble sleeping, diarrhoea, constipation, pyrexia, anemia, edemia, dyspnea, rash, abdominal pain, myalgia, dehydration, hypotension, anziety and so on,. To overcome these problems, bortezomib analogues are targeted [18]. The peptide based vaccine Nano vaccine adjuvants, and aromatic peptides and beta amino acids are better tool of cancer treatment [1925].

Cancer is one of the most dangerous diseases caused by mutation in genetic material. Malignant cells are more susceptible to proteasome inhibition than normal cells is the key to achieve a suitable therapeutic ratio. Bortezomib is the first proteasome inhibitor in multiple myeloma and mantle cell Lymphoma who have received at least one prior therapy. But continuous dosage leads to few side effects and overcome by targeted bortezomib analogues.

\section{References}

1. Paramore A, Frantz A (2003) Bortezomib. Nat Rev Drug Discov 2: 611-612.

2. Richardson PG, Hideshima T, Anderson KC (2003) Bortezomib (PS-341): a novel, first-in-class proteasome inhibitor for the treatment of multiple myeloma and other cancers. Cancer Control 10: 361-369.

3. Voorhees PM, Orlowski RZ (2006) The proteasome and proteasome inhibitors in cancer therapy. Annu Rev Pharmacol Toxicol 46: 189-213.

4. Adams J (2004) The proteasome: a suitable antineoplastic target. Nat Rev Cancer 4: 349-360.

5. Rajkumar SV, Richardson PG, Hideshima T, Anderson KC (2005) Proteasome inhibition as a novel therapeutic target in human cancer. $\mathrm{J}$ Clin Oncol 23: 630-639.

6. Richardson PG, Mitsiades C, Hideshima T, Anderson KC (2005) Proteasome inhibition in the treatment of cancer. Cell Cycle 4: 290-296.

7. Groll M, Huber R, Moroder L (2009) The persisting challenge of selective and specific proteasome inhibition. J Pept Sci 15: 58-66.

8. Zhu YQ, Lei M, Lu AJ, Zhao X, Yin XJ, et al. (2009) 3D-QSAR studies of boron-containing dipeptides as proteasome inhibitors with CoMFA and CoMSIA methods. 44: 1486-1499.

9. Myung J, Kim KB, Crews CM (2001) The ubiquitin-proteasome pathway and proteasome inhibitors. Med Res Rev 21: 245-273.

10. Ludwig H, Khayat D, Giaccone G, Facon T (2005) Proteasome inhibition and its clinical prospects in the treatment of hematologic and solid malignancies. Cancer 104: 1794-1807.

11. Tian B, Brasier AR (2003) Identification of a nuclear factor kappa B-dependent gene network. Recent Prog Horm Res 58: 95-130.

12. Hideshima T, Richardson $P$, Chauhan D, Palombella VJ, Elliott PJ, et al. (2003) The proteasome inhibitor PS-341 inhibits growth, induces apoptosis, and overcomes drug resistance in human multiple myeloma cells. Cancer Research 61: 3071-3076.

13. Sunwoo JB, Chen Z, Dong G, Yeh N, Crowl Bancroft C, et al. (2001) Novel proteasome inhibitor PS-341 inhibits activation of nuclear factor-kappa B, cell survival, tumor growth, and angiogenesis in squamous cell carcinoma. Clin Cancer Res 7: 1419-1428.

14. Zhang HG, Wang J, Yang X, Hsu HC, Mountz JD (2004) Regulation of apoptosis proteins in cancer cells by ubiquitin. Oncogene 23: 2009-2015.

15. Cross B, Chen L, Cheng Q, Li B, Yuan ZM, et al. (2011) Inhibition of p53 DNA binding function by the MDM2 protein acidic domain. J Biol Chem 286: 16018-16029.

16. Oakervee HE, Popat R, Curry N, Smith P, Morris C, et al. (2005) PAD combination therapy (PS-341/bortezomib, doxorubicin and dexamethasone) for previously untreated patients with multiple myeloma. $\mathrm{Br} \mathrm{J}$ Haematol 129: 755-762.

17. Chen D , Frezza M, Schmitt S, Kanwar J, Dou QP (2011) Bortezomib as the first proteasome inhibitor anticancer drug: current status and future perspectives. Curr Cancer Drug Targets 11: 239-253.

18. Field-Smith A1, Morgan GJ, Davies FE (2006) Bortezomib (Velcadetrade mark) in the Treatment of Multiple Myeloma. Ther Clin Risk Manag 2: 271 279.

19. Rajagopal A, Aravinda S, Raghothama S, Shamala N, Balaram P (2011) Chain length effects on helix-hairpin distribution in short peptides with AidDAla and Aib-Aib Segments. Biopolymers 96: 744-756.

20. Raghavender US, Chatterjee B, Saha I, Rajagopal A, Shamala N, et al. (2011) Entrapement of a Water Wire in a Hydrophobic Peptide Channel with an Aromatic Lining. J Phys Chem B 115: 9236-9243.

21. Rajagopal A, Aravinda S, Raghothama S, Shamala N, Balaram P (2012) 
Aromatic interactions in model peptide $\beta$-hairpins: Ring current effects on proton chemical shifts. Biopolymers 98: 185-194.

22. Basuroy K, Rajagopal A, Raghothama S, Shamala N, Balaram P (2012) $\beta$-Turn Analogues in Model $\alpha \beta$-Hybrid Peptides: Structural Characterization of Peptides containing $\beta 2.2 A c 6 c$ and $\beta 3.3 A c 6 c$ Residues. Chem Asian J 7: 1671.

23. Rajagoapl A, Charles BC, Alexey YK, Joshua DS, Frederick JK, et al. (2015)
Enhancing the Magnitude of Antibody Responses through Biomaterial Stereochemistry. ACS Biomater. Sci. Eng 1: 601-609.

24. Koyfman AY, Appavu R, Sheller S, Rudra JS (2015) Self-Assembly of Heterochiral Peptides with Varied Sequence Patterns. 1.

25. Rudra JS, Ding Y, Neelakantan H, Ding C, Appavu R, et al. (2016) Suppression of Cocaine-evoked Hyperactivity by Self adjuvanting and Multivalent Peptide Nanofiber Vaccines. ACS Chem Neurosci. 